\title{
ALGEBRAIC COBORDISM OF SIMPLY CONNECTED LIE GROUPS
}

\author{
NOBUAKI YAGITA
}

\begin{abstract}
Let $G_{\mathbb{C}}$ be the algebraic group over $\mathbb{C}$ corresponding a simply connected Lie group $G$. The algebraic cobordism $\Omega\left(G_{\mathbb{C}}\right)$ defined by Levine and Morel is showed isomorphic to $M U^{*}$-subalgebra of $M U^{*}(G)$ with some modulous and is computed explicitely.
\end{abstract}

\section{INTRODUCTION}

For a smooth algebraic variety $X$ over a field $k$ of $\operatorname{ch}(k)=0$, recently Levine and Morel [L-M 1,2] defined the algebraic cobordism $\Omega^{*}(X)$ such that $\Omega^{*}(X) \otimes_{\Omega^{*}} Z \cong C H^{*}(X)$; the Chow ring of $X$ and that there are maps

$$
\Omega^{*}(X) \stackrel{\rho_{M G L}}{\longrightarrow} M G L^{2 *, *}(X) \stackrel{t_{M U}}{\longrightarrow} M U^{*}(X)
$$

where $M G L^{2 *, *}(-)=\oplus_{i} M G L^{2 i, i}(-)$ is the motivic cobordism theory defined by Voevodsky [V 1] and $M U^{*}(-)$ is the usual complex cobordism theory.

Let $G$ be a simply connected Lie group and $G_{\mathbb{C}}$ the corresponding reductive algebraic group over $\mathbb{C}$; the complex number field. Let $T$ be the maximal torus of $G$ and $\pi: G \rightarrow G / T$ is the projection. By Grothendieck and $\mathrm{Kac}[\mathrm{K}]$, it is known

$$
C H^{*}\left(G_{\mathbb{C}}\right) \cong \pi^{*}\left(H^{*}(G / T)\right) \text {. }
$$

We study the cobordism version of this result. Fix a prime $p$. Recall $\Omega^{*} \cong M U^{*} \cong \mathbb{Z}\left[x_{1}, \ldots\right]$ with $\left|x_{i}\right|=-2 i$ and identify $v_{i}=x_{p^{i}-1}$.

Theorem 1.1. Let $I=\left(p, v_{1}, \ldots\right)$ be the invariant prime ideal of $M U^{*}$. Then we have the isomorphism $\Omega^{*}\left(G_{\mathbb{C}}\right) / I^{2} \cong \pi^{*}\left(M U^{*}(G / T)\right) / I^{2}$.

The above isomorphism seems to hold without $I^{2}$, however we can not prove it now.

By Borel theorem, we can write $H^{*}(G ; \mathbb{Z} / p) \cong P\left(y_{\text {even }}\right) / p \otimes \Lambda\left(x_{\text {odd }}\right)$ where $P\left(y_{\text {even }}\right)$ is a truncated polynomial algebra of even degree generators $y_{\text {even }}$ and $\Lambda\left(x_{\text {odd }}\right)$ is the exterior algebra of odd degree generators $x_{\text {odd }}$. When $p=2$ we take $y_{\text {even }}$ as a power of some $x_{\text {odd }}$. Then the result of Grothendieck and $\mathrm{Kac}[\mathrm{K}]$ is stated as

$$
C H^{*}\left(G_{\mathbb{C}}\right)_{(p)} \cong P\left(y_{\text {even }}\right) / p \text {. }
$$

Let $Q_{i}$ be the Milnor primitive operation inductively defined by $Q_{i}=\left[Q_{i-1}, P^{p^{i-1}}\right]$ and $Q_{0}=\beta$; the Bockstein operation. It is known that $Q_{i}\left(x_{\text {odd }}\right) \in P\left(y_{\text {even }}\right) / p$ for all $i \geq 0$.

Theorem 1.2. There is an $\Omega^{*}$-algebra isomorphism

$$
\Omega^{*}\left(G_{\mathbb{C}}\right) / I^{2} \cong \Omega^{*} \otimes P\left(y_{\text {even }}\right) /\left(I^{2}, \sum_{i} v_{i} Q_{i}\left(x_{\text {odd }}\right)\right) .
$$

1991 Mathematics Subject Classification. Primary 55P35, 57T25; Secondary 55R35, 57T05.

Key words and phrases. algebraic cobordism, Chow ring, motivic cohomology, BP-theory, simply connected Lie groups. 
For all simply connected simple Lie groups, more explicit forms of this theorem are shown in Theorem 5.1-5.4.

As for motivic cobordism theory $M G L^{*, *}\left(G_{\mathbb{C}}\right)$, we do not know well. Indeed, even $M U^{*}(G)_{(p)}$ are quite complicated and unknown, in general. Let $M G L / p^{*, *}$ be the $\bmod$ $p$ motivic cobordism theory defined by the cofibering

$$
M G L \stackrel{p}{\longrightarrow} M G L \rightarrow M G L / p
$$

in the stable $\mathbb{A}^{1}$-homotopy category. Let $M U / p^{*}(X)=M U^{*}(-; \mathbb{Z} / p)$ the $\bmod p$ complex cobordism theory defined similarly.

Theorem 1.3. Let $G$ be a simle Lie group in Case I in $\S 5$, e.g., $G=F_{4}, E_{6}, E_{7}$ for $p=3$. Then we can give bidegree to $M U / p^{*}(G)$ such that degree of its $M U^{*}$-algebra generators are $(2 n, n)$ or $(2 m-1, m)$ for $n, m \geq 3$, and that

$$
M U / p^{*}(G) \subset M G L / p^{*, *}\left(G_{\mathbb{C}}\right) .
$$

In $\S 2$, we recall the definition of the algebraic cobordism $\Omega^{*}(X)$ and the relation to the complex cobordism $M U^{*}(X)$. We also remark the $B P$-version of $\Omega^{*}(X)$. In $\S 3$, we note that Theorem 1.1 holds if $\pi^{*} M U^{*}(G / T) \cong M U^{*}(G / T) / \operatorname{Ideal}\left(i^{*} \tilde{M}^{*}(B T)\right)$. Here

$$
G \stackrel{\pi}{\longrightarrow} G / T \stackrel{i}{\longrightarrow} B T .
$$

is the usual fibering. In $\S 4$, we will prove the above isomorphism $\bmod \left(I^{2}\right)$ with some assumption of the Milnor operation $Q_{i}$ on $H^{*}(G ; \mathbb{Z} / p)$. Here we use the spectral sequence for connected Morava $K$-theory (or other cohomology theories)

$$
E_{2}^{*, *} \cong H^{*}\left(B T ; k(1)^{*}(G)\right) \Longrightarrow k(1)^{*}(G / T) .
$$

Indeed, $E_{\infty}^{*, 0}$ shows the image $i^{*} k(1)^{*}(B T)$. In $\S 5$, we check the condition of $Q_{i}$, for each simple Lie group which has $p$-torsion, and consequently we see Theorem 1.1. Explicit results of $\Omega^{*}\left(G_{\mathbb{C}}\right) / I^{2}$ also given here. In $\S 6$, we give examples of computations of the above spectral sequence for the easy cases $G$. In $\S 7$, for this type group $G$, we also study the motivic cobordism $M G L / p^{*, *}\left(G_{\mathbb{C}}\right)$. The last section is very short remark for classifying spaces $B G$.

\section{ALGEBRAIC COBORDISM}

By extending the arguments by Quillen [Q], Levine and Morel defined the algebraic cobordism theory $\Omega^{*}(-)$ as the universal theory in theories having transfers and Chern classes [L-M 1,2] ( We say that $h^{*}(X)$ is a theory having transfers and Chern classes if this theory satifies the actioms A1 to A4 in [L-M 1]). Here we note that $\Omega^{*}(-)$ is not cohomology theory. The ring $\Omega^{*}(X)$ is constructed as

$$
\left.\Omega^{*}(X)=\{[f: M \rightarrow X]\} / \text { (relations }\right) .
$$

Here $f$ is a map from a smooth variety $M$ to $X$ of pure codimension, namely, $\operatorname{dim}_{f(y)}(X)-$ $\operatorname{dim}_{y}(M)$ is constant for all $y$ in the same connected component of $M$. Relations are given so that we can define Chern classes or formal group laws ( for details, see [L-M 1]). Given theory $h^{*}(-)$ having transfers and Chern classes, the map

$$
\rho_{h}: \Omega^{*}(-) \rightarrow h^{*}(-)
$$

is defined by $\rho_{h}([f: M \rightarrow X])=f_{*}\left(1_{M}\right)$ where $1_{M} \in h^{0}(M)$ represents the identity element.

Let $H^{*, *}(-)$ (resp. $M G L^{*, *}(-), M U^{*}(-), C H^{*}(-), H^{*}(-)$ ) be the motivic cohomology theory defined by Suslin and Voevodsky (resp. the motivic cobordism theory, the complex 
cobordism theory, the Chow ring, the usual cohomology theory). Then we have commutaive diagram

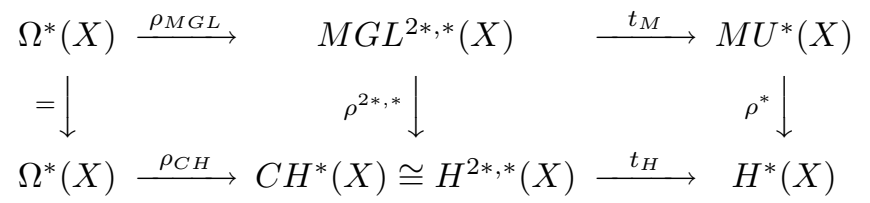

where $\rho^{2 *, *}, \rho^{*}$ (resp. $\left.t_{m}, t_{H}\right)$ are Thom maps (resp. realization maps.) Levine and Morel proves that

$$
\begin{gathered}
t_{M} \rho_{M G L}: \Omega^{*}(p t) \cong M U^{*}(p t) \cong \mathbb{Z}\left[x_{1}, x_{2}, \ldots\right] \text { with } \quad\left|x_{i}\right|=-2 i \\
\text { and } \rho_{C H} \otimes_{\Omega^{*}} Z: \Omega^{*}(X) \otimes_{\Omega^{*}} Z \cong C H^{*}(X) .
\end{gathered}
$$

Moreover they conjecture that $\rho_{M G L}$ are always isomorphisms.

For the loaclized theories $h^{*}(-)_{(p)}$, we can consider the $B P$-version by using the universal $p$-typical formal group laws. However we construct here the $B P$-version using the Novikov's technique (5.4 in [N]). Given a sequence $\alpha=\left(a_{1}, a_{2}, ..\right), a_{i} \geq 0$, recall the Landweber-Novikov operation is defined by

$$
S_{\alpha}\left(f_{*}\left(1_{M}\right)\right)=f_{*}\left(c_{\alpha}\left(N_{f}\right)\right) \quad \text { for }[f: M \rightarrow X] \in \Omega^{*}(X)
$$

where $c_{\alpha}\left(N_{f}\right)$ is the Chern class of the normal bundle of $f(M)$ in $X$ so that $S_{\alpha}$ in $M U^{*}(X)$ is the usual Landweber-Novikov operation. This operations satisfy the Cartan formula $S_{\alpha}(x y)=\Sigma_{\alpha=\beta+\gamma} S_{\beta}(x) S_{\gamma}(y)$ for $x, y \in \Omega^{*}(X)$. Define an operation

$$
\Delta_{x_{i}}=\Sigma_{q \geq 1}\left(x_{i} / S_{\Delta_{i}}\left(x_{i}\right)\right)^{q-1} S_{q \Delta_{i}} .
$$

Note that $\Delta_{x_{i}}\left(x_{i}\right)=1$ and $S_{\Delta_{i}}\left(x_{i}\right) \neq 0 \bmod p$ if $i \neq p^{j}-1$. Then we can easily prove that $\pi_{i}=1-x_{i} \Delta_{x_{i}}$ is a multiplicative projection such that $\pi_{i}\left(x_{j}\right)=\left(1-\delta_{i j}\right) x_{j}$. Essentially composing (for details, see p587 in [N]) $\pi_{i}$ for all $i \neq p^{j}-1$, we get the multiplicative projection $\Phi: \Omega^{*}(-)_{(p)} \rightarrow \Omega^{*}(-)_{(p)}$ such that

$$
\Phi\left(x_{i}\right)= \begin{cases}x_{i} & \left(\text { if } i=p^{j}-1 \text { for some } j\right) \\ 0 & \text { (otherwise })\end{cases}
$$

Define the algebraic Brown-Peterson theory

$$
\Omega_{B P}^{*}(X)=\operatorname{Im}\left(\Phi\left(\Omega^{*}(X)_{(p)}\right) \subset \Omega^{*}(X)_{(p)} .\right.
$$

Hence if $h^{*}(-)$ is a theory having transfers and Chern classes, then there is the natural map $\rho_{B P, h}: \Omega_{B P}^{*}(X) \rightarrow h^{*}(X)_{(p)}$ compatible with $\rho_{h(p)}$.

Lemma 2.1. Identifying $\Omega_{B P}^{*}=M U_{(p)}^{*} /\left(x_{i} \mid i \neq p^{j}-1\right)$, we have the isomorphism $\Omega_{B P}^{*}(X) \cong$ $\Omega_{B P}^{*} \otimes_{\Omega_{(p)}^{*}} \Omega^{*}(X)_{(p)}$

Proof. Since $\pi_{x_{i}}(a)=\left(1-x_{i} \Delta_{x_{i}}\right) a=a \bmod \left(x_{i}\right)$, we see $\Phi(a)=a \bmod \left(x_{i} \mid i \neq p^{j}-1\right)$ for all $a \in \Omega^{*}(X)_{(p)}$.

In this paper we mainly consider $\Omega^{*}(X)_{(p)}$ but not $\Omega_{B P}^{*}(X)$. However we use notations $v_{i}=x_{p^{i}-1} \in \Omega_{(p)}^{*}$ for ease of expressions. 


\section{LIE GROUP AND ITS MAXIMAL TORUS}

Let $G$ be a simply connected Lie group and $G_{\mathbb{C}}$ the corresponding reductive algebraic group over $\mathbb{C}$. Let $T$ be the maximal torus of $G$. then we have the fibering

$$
G \stackrel{\pi}{\longrightarrow} G / T \stackrel{i}{\longrightarrow} B T=E T / T
$$

Taking $B T=\operatorname{colim}_{N \rightarrow \infty}\left(\left(\mathbb{A}^{N}-\{0\}\right)^{\times n} /\left(\mathbb{G}_{m}\right)^{n}\right)$, we get the maps of algebraic groups

$$
G_{\mathbb{C}} \stackrel{\pi_{\mathbb{C}}}{\longrightarrow} G_{\mathbb{C}} / T_{\mathbb{C}} \stackrel{i_{\mathbb{C}}}{\longrightarrow} B T
$$

If an algebraic variety $X$ has a cellular decomposition,i.e., $X=X_{n} \supset \ldots \supset X_{0}$ with $X_{i}-X_{i-1}=\cup \mathbb{A}^{n_{i j}}$ where $\mathbb{A}^{n_{i j}}$ is the affine space of $\operatorname{dim}=n_{i j}$, then the Chow ring is isomorphic to the ordinary cohomology i.e., $C H^{*}(X) \cong H^{*}(X)$ and moreover $M U^{*}(X) \cong$ $M U^{*} \otimes H^{*}(X)$. Hence these facts hold for $X=G_{\mathbb{C}}$ and $B T_{\mathbb{C}}$.

From Grothendieck and Kac $[\mathrm{K}]$, it is known that

$$
C H^{*}\left(G_{\mathbb{C}}\right) \cong \operatorname{Im}\left(\pi^{*}: H^{*}(G / T) \rightarrow H^{*}(G)\right)
$$

Lemma 3.1. Let $\tilde{M} U^{*}(-)$ be the reduced $M U^{*}(-)$ theory. If there is the isomorphism

$$
\pi^{*}\left(M U^{*}(G / T)\right) \cong M U^{*}(G / T) /\left(\operatorname{Ideal}\left(i^{*} \tilde{M} U^{*}(B T)\right)\right.
$$

then we have

$$
M G L^{2 *, *}\left(G_{\mathbb{C}}\right) \supset \Omega^{*}\left(G_{\mathbb{C}}\right) \cong \pi^{*}\left(M U^{*}(G / T)\right)
$$

Proof. Consider the diagram

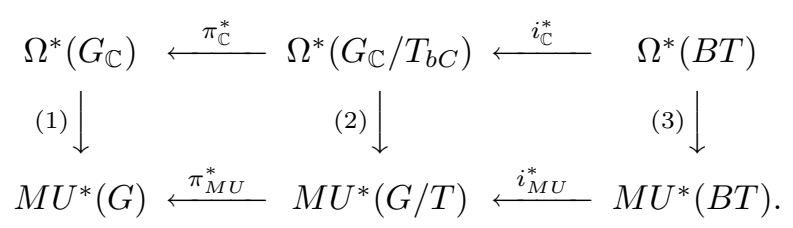

where (1),(2),(3) are maps induced from $t_{M U} \rho_{M G L}$. For $X=G / T, B T$, we already know that $M U^{*}(X) \cong M U^{*} \otimes H^{*}(X) \cong M U^{*} \otimes C H^{*}(X)$. Hence (2) and (3) are isomorphisms. While the rows are not exact, we get the map

$$
\Omega^{*}\left(G_{\mathbb{C}}\right) \stackrel{\pi^{*}}{\longleftarrow} M U^{*}(G / T) /\left(\operatorname{Ideal}\left(i_{M U}^{*} \tilde{M} U^{*}(B T)\right)\right)
$$

where $\pi^{*}$ is induced from $\pi_{\mathbb{C}}^{*}(2)^{-1}$. Tensoring $\otimes_{\Omega^{*}} \mathbb{Z}$, we have

$$
\begin{aligned}
& C H^{*}(G) \cong \Omega^{*}(G) \otimes_{\Omega^{*}} \mathbb{Z} \quad \pi^{*} \stackrel{\otimes}{M U}^{*} \mathbb{Z} \\
&\left(M U^{*}(G / T) /\left(\operatorname{Ideal}\left(i_{M U}^{*}\right)\right) \otimes_{M U} * \mathbb{Z} \cong H^{*}(G / T) /\left(\operatorname{Ideal}\left(i_{H}^{*}\right)\right) .\right.
\end{aligned}
$$

By Grothendieck and Kac theorem the above map is epic. Hence $\pi^{*}$ itself also epic. From the assumption of this lemma, we get $\operatorname{Im}\left(\pi_{M U}^{*}\right) \cong M U^{*}(G / T) /\left(\operatorname{Ideal}\left(i_{M U}^{*}\right)\right)$. Hence the $\operatorname{map} \pi^{*}$ is an isomorphism.

Since $(1)=t_{M U} \rho_{M G L}$ is injective, the map $\rho_{M G L}$ is a split injection. 


\section{4. $B P^{*}(G / T) / \operatorname{Ideal}\left(i^{*}\left(\tilde{B P}{ }^{*}(B T)\right)\right.$}

In this section, we consider $\Omega_{B P}^{*}\left(G_{\mathbb{C}}\right)$ or $\Omega^{*}\left(G_{\mathbb{C}}\right)_{(p)}$. Moreover we study $B P^{*}(G)$ instead of $M U^{*}(G)_{(p)}$. Let $G$ be a simply connected Lie group. By the Borel's therorem, we have the ring isomorphism for $p$ odd

$$
H^{*}(G ; \mathbb{Z} / p) \cong P(y) / p \otimes \Lambda\left(x_{1}, \ldots, x_{l}\right) \quad \text { with } P(y)=\mathbb{Z}\left[y_{1}, \ldots, y_{k}\right] /\left(y_{1}^{p^{r_{1}}}, \ldots y_{k}^{p^{r_{k}}}\right)
$$

where $\left|y_{i}\right|=$ even and $\left|x_{j}\right|=o d d$. When $p=2$, the righthand side is isomorphic to $\operatorname{grH}^{*}(G ; Z / 2)$ and for each $y_{i}$, there is $x_{j}$ with $x_{j}^{2}=y_{i}$. By Grothendieck and Kac $[\mathrm{K}]$, we know that

$$
C H^{*}\left(G_{\mathbb{C}}\right) / p \cong P(y) / p .
$$

Consider the spectral sequence induced from the cofibering (3.1)

$$
E_{2}^{*, *}=H^{*}\left(B T ; H^{*}(G ; \mathbb{Z} / p)\right) \Longrightarrow H^{*}(G / T ; \mathbb{Z} / p) .
$$

The cohomology of the classifying space of the torus is

$$
H^{*}(B T) \cong \mathbb{Z}\left[t_{1}, \ldots, t_{l}\right] \quad \text { with } \quad\left|t_{i}\right|=2
$$

where $l$ is also the number of the odd degree generatos $x_{k}$ in $H^{*}(G ; \mathbb{Z} / p)$. It is known that there is a regular sequence $\left(b_{1}, \ldots, b_{l}\right)$ in $H^{*}(B T) / p$ such that $d_{\left|x_{i}\right|+1}\left(x_{i}\right)=b_{i}$. Thus we get

$$
\text { (4.4) } \quad H^{*}(G / T ; \mathbb{Z} / p) \cong P(y) \otimes \mathbb{Z} / p\left[t_{1}, \ldots, t_{l}\right] /\left(b_{1}, \ldots, b_{l}\right) .
$$

Recall the connected Morava K-theory $k(n)^{*}(-)$ with the coefficient $k(n)^{*}=Z / p\left[v_{n}\right]$. Then the usual Morava $K$-theory is $K(n)^{*}(X)=\left[v_{n}^{-1}\right] k(n)^{*}(X)$.

Since $H^{*}(G / T)$ has no torsion, we get the $B P^{*}$-modules isomorphism

$$
B P^{*}(G / T) \cong B P^{*} \otimes P(y) \otimes \mathbb{Z}\left[t_{1}, \ldots, t_{l}\right] /\left(\tilde{b}_{1}, \ldots, \tilde{b}_{l}\right)
$$

where $\tilde{b}_{i}=b_{i} \bmod (p)$. However note that this is not a $B P^{*}$-algebras isomorphism. (The righthand side above is isomorphic to $\operatorname{gr} B P^{*}(G / T)$.) We also note $k(n)^{*}(G / T) \cong k(n)^{*} \otimes_{B P^{*}}$ $B P^{*}(G / T)$ and

$$
k(n)^{*}(G / T) \cong k(n)^{*} \otimes P(y) \otimes \mathbb{Z} / p\left[t_{1}, \ldots, t_{l}\right] /\left(b_{1}, \ldots, b_{l}\right) .
$$

We want to study the spectral sequence

$$
\text { (4.7) } \quad E_{2}^{*, *}=H^{*}\left(B T ; k(1)^{*}(G)\right) \Longrightarrow k(1)^{*}(G) .
$$

Let $\rho: k(n)^{*}(X) \rightarrow H^{*}(X ; \mathbb{Z} / p)$ be the natural (Thom) map. Let us write by $X^{s}$ the $s$-skeleton of $X$. Recall also $Q_{i}$ is the Milnor primitive operation inductively defined by $Q_{i}=P^{p^{i-1}} Q_{i-1}-Q_{i-1} P^{p^{i-1}}$ where $P^{k}$ are reduced power operations and $Q_{0}=\beta$; Bockstein operation. Here we recall lemmas related about relations between $B P^{*}$-modules structure of $B P^{*}(X)$ and $Q_{i}$-acotions on $H^{*}(X ; \mathbb{Z} / p)$.

Lemma 4.1. ([Y 1]) Let $\sum_{n} v_{n} y_{n}=0 \in B P^{*}(X)$. Then there is $x \in H^{*}(X ; \mathbb{Z} / p)$ such that $Q_{n}(x)=\rho\left(y_{n}\right)$.

Lemma 4.2. For $x \in H^{*}(X ; \mathbb{Z} / p)$, there is $y^{\prime} \in k(n)^{*}(X)$ with $Q_{n}(x)=\rho\left(y^{\prime}\right)$ and $v_{n} y^{\prime}=0$. Conversely if $v_{n} y^{\prime}=0$, then there is $x$ with $Q_{n}(x)=\rho\left(y^{\prime}\right)$.

Proof. There is the Sullivan-Bockstein exact sequence

$$
k(n)^{*}(X) \stackrel{v_{n}}{\longrightarrow} k(n)^{*}(X) \stackrel{\rho}{\longrightarrow} H^{*}(X ; \mathbb{Z} / p) \stackrel{\delta}{\longrightarrow} k(n)^{*}(X) \stackrel{v_{n}}{\longrightarrow}
$$

Since $\rho \delta=Q_{n}$, if $Q_{n} x=y$, then $\delta(x)=y^{\prime}$ is $v_{n}$-torsion. Conversely if $v_{n} y^{\prime}=0$, then there is $x \in H^{*}(X ; \mathbb{Z} / p)$ with $\delta(x)=y^{\prime}$ and $Q_{n}(x)=\rho\left(y^{\prime}\right)$. 
Lemma 4.3. Let $F \rightarrow E \stackrel{p}{\longrightarrow} B$ be a fiber bundle such that $H^{*}(B)$ is a p-torion free simply connected space. In the Serre spectral sequence converging to $H^{*}(E ; \mathbb{Z} / p)$, let $0 \neq$ $y \in H^{*}(F ; \mathbb{Z} / p)$ and $0 \neq b \in H^{*}(B ; \mathbb{Z} / p)$ such that if $Q_{n} x=y$, then $d_{r}(x)=b$ and that there exists such $x \in H^{*}(F ; \mathbb{Z} / p)$. Then there are $y^{\prime} \in k(n)^{*}(F)$ and $b^{\prime} \in k(n)^{*}(B)$ such that $\rho\left(y^{\prime}\right)=y, \rho\left(b^{\prime}\right)=b$ and

$$
v_{n} y^{\prime}=\lambda b^{\prime}, \quad \lambda \neq 0 \in \mathbb{Z} / p \quad \text { in } k(n)^{*}\left(p^{-1} B^{|b|}\right)
$$

Proof. Let $B^{\prime}=B^{|b|-1}$ and $E^{\prime}=p^{-1}\left(B^{\prime}\right)$. Consider the Serre spectral sequence

$$
E_{2}^{*, *}=H^{*}\left(B^{\prime} ; H^{*}(F ; \mathbb{Z} / p)\right) \Longrightarrow H^{*}\left(E^{\prime} ; \mathbb{Z} / p\right) \text {. }
$$

Since $d_{r} x=b=0 \in H^{*}\left(B^{\prime}\right)$, there is $x \in H^{*}\left(E^{\prime} ; Z / p\right)$ with $Q_{n} x=y$. This means there exists $v_{n} y^{\prime}=0$ in $k(n)^{*}\left(E^{\prime}\right)$.

On the otherhand, let $B^{\prime \prime}=B^{|b|-1} \cup e_{b}$ and $E^{\prime \prime}=p^{-1} B^{\prime \prime}$ where $e_{b}$ is the normal cell representing $b$. Then $d_{r} x=b \neq 0$ and there is no element $x \in H^{*}\left(E^{\prime \prime} ; \mathbb{Z} / p\right)$ with $Q_{n} x=\rho(y)$. From Lemma 4.2, we get $v_{n} y^{\prime} \neq 0 \in k(n)^{*}\left(E^{\prime \prime}\right)$ and have this lemma.

Remark (4.8). Lemma 4.1 and Lemma 4.2 are also hold for $n=0$ replacing $k(0)^{*}(-)=$ $H^{*}(-)$ : the integral cohomology and $v_{0}=p, \lambda \neq 0 \bmod (p)$.

The $Q_{i}$ actions on $H^{*}(G ; \mathbb{Z} / p)$ are quite restrictive. We consider the following assumption.

Assumption (4.9)

(A.1) if $i \neq j$, then $\left|x_{i}\right| \neq\left|x_{j}\right|$.

(A.2) $Q_{k} x_{i} \in P(y)$ for all $k, i$ and if $Q_{k} x_{i} \neq 0$, then $Q_{0} x_{i}=y_{i}$ or, $Q_{0} x_{i}=0$ and $Q_{1} x_{i}=y_{i^{\prime \prime}}$ for some $i^{\prime \prime}$.

The above assumptions are satisfied for all simply connected simple Lie groups except for $p=2, \operatorname{Spin}(n), E_{8}$. However quite similar conditions also hold for these cases. (We will expain these in the next section.)

Lemma 4.4. Assume $(A, 1),(A .2)$. Then $\left.B P^{*}(G / T) / \operatorname{Ideal}\left(i^{*}\left(\tilde{B P^{*}}(B T)\right), I^{2}\right)\right)$ is a quotient ring of $B P^{*} \otimes P(y) /\left(I^{2},(1),(2)\right)$ where

$$
\begin{aligned}
(1) \quad p y_{i}=0 \quad \bmod \left(v_{1}, \ldots\right) \quad \text { if } Q_{0} x_{i}=y_{i} \\
\text { (2) } v_{1} y_{j}=0 \bmod \left(v_{2}, \ldots\right) \text { if } Q_{1} x_{j^{\prime}}=y_{j} \text { but } Q_{0} x_{j^{\prime}}=0 .
\end{aligned}
$$

Proof. First note that from (4.5), the $B P^{*}$-algebra is a quotient of $B P^{*} \otimes P(y)$. Recall in the spectral sequence (4.3), $d_{r}\left(x_{i}\right)=b_{i}$ and $Q_{0}\left(x_{i}\right)=y_{i}$. Letting $F=G, G / T=E, B=B T$, from Remark (4.8), we know

$$
p y_{i}=i^{*}\left(b_{i}\right) \quad \text { in } H^{*}(G / T)=B P^{*}(G / T) /\left(v_{1}, v_{2}, \ldots\right) .
$$

From (4.5), we have $p y_{i}+\sum v_{n} a_{n} \in \operatorname{Ideal}\left(i^{*}\left(\tilde{B P^{*}}(B T)\right)\right.$ for $a_{n} \in B P^{*} \otimes P(y)$. Hence the relation (1) containd in $\operatorname{Ideal}\left(i^{*}\left(\tilde{B P}^{*}(B T)\right)\right.$.

Let $Q_{1} x_{i^{\prime}}=y_{i}$. Recall $b_{i^{\prime}}=d_{\left|x_{i^{\prime}}\right|+1}\left(x_{i^{\prime}}\right)$ in the spectral sequence (4.3). From Lemma 4.3 , we already know in $k(1)^{*}(G / T)$

$$
(*) \quad v_{1} y_{i}=\lambda b_{i^{\prime}}+b^{\prime} \quad \lambda \neq 0 \in \mathbb{Z} / p, b^{\prime} \in E_{\infty}^{\left|b_{i^{\prime}}\right|+1, *} .
$$

Since $b_{i^{\prime}}=0$ in $H^{*}(G / T ; \mathbb{Z} / p)$, we know $b^{\prime}=0 \bmod \left(v_{1}\right)$ in $k(1)^{*}(G / T)$. Here we recall all elements are represented as in (4.7). Hence we can write

$$
b^{\prime}=v_{1} d(y)+v_{1} g \quad \text { with } \quad d(y) \in P(y), g \in I(t)=\operatorname{Ideal}\left(t_{1}, \ldots, t_{l}\right) \subset k(1)^{*}(G / T) .
$$

Let $E^{\prime}=p^{-1} G / T^{\left|b_{i^{\prime}}\right|}$. Since $b^{\prime} \in E_{\infty}^{\left|b_{i^{\prime}}\right|+1, *}$, we see $b^{\prime}=0 \in k(1)^{*}\left(E^{\prime}\right)$. Hence there is $x \in H^{*}\left(E^{\prime} ; \mathbb{Z} / p\right)$ such that $Q_{1}(x)=b^{\prime} / v_{1}=d(y)+g$. But if $b^{\prime} \neq 0 \in k(1)^{*}(G / T)$, then $x$ does not exist in $H^{*}(G / T ; \mathbb{Z} / p)$, namely, the corresponding element $x$ in $E_{2}^{* * *}$ of $(4.7)$ is not 
a permanent cycle. In particular if $d(y) \neq 0$, then $x \neq 0 \in H^{*}(G ; \mathbb{Z} / p)$ but $|x|=\left|x_{i^{\prime}}\right|$ and there is no differential $d_{r}(x) \neq 0$ for $r>\left|x_{i^{\prime}}\right|+1=\left|b_{i^{\prime}}\right|$; this is a contradiction. Hence we get $b^{\prime} \in I(t)$ and so $v_{1} y_{i} \in I(t)$.

Let $Q_{0} x_{i^{\prime}}=0$ and $Q_{1} x_{i^{\prime}}=y_{i}$. From $\left(^{*}\right)$, we get in $B P^{*}(G / T)$,

$$
v_{1} y_{i}=\lambda \tilde{b}_{i^{\prime}}+\tilde{b}^{\prime}+p f(y)+p b^{\prime \prime} \bmod \left(p^{2}, v_{2}, v_{3}, \ldots\right)
$$

where $\tilde{b}_{i^{\prime}}=b_{i^{\prime}} \bmod (p), \tilde{b}^{\prime}=b^{\prime} \bmod (p), f(y) \in P(y)$ and $b^{\prime \prime} \in \operatorname{Ideal}\left(t_{1}, \ldots, t_{l}\right) \subset B P^{*}(G / T)$. But $f(y)=0 \bmod \left(p^{2}\right)$ otherwise $Q_{0} x_{i^{\prime}}=-f(y) \neq 0$ from Remark 4.8. . Thus we get (2) is contained in $\operatorname{Ideal}\left(i^{*}\left(\tilde{B P^{*}}(B T)\right)\right.$.

Lemma 4.5. Assume (A,2). The image $\pi^{*} B P^{*}(G / T) / I^{2}$ is isomorphic to

$$
B P^{*} \otimes P(y) /\left(I^{2},(1),(2) \text { in Lemma } 4.3\right) \text {. }
$$

Proof. Suppose that there is a relation in $\pi^{*} B P^{*}(G / T) / I^{2}$

$$
(*) \quad v_{n} y=0 \quad \bmod \left(I^{2}, v_{n+1}, \ldots\right) \quad \text { with } y \in P(y) .
$$

From Lemma 4.1, there is $x \in H^{*}(G ; \mathbb{Z} / p)$ such that $Q_{n} x=y$ and $Q_{j}(x)=0 \bmod \left(I^{2}\right)$ for $j<n$. Let $H^{*}(G ; \mathbb{Z} / p)=\oplus_{s} X_{s}$ where $X_{s}$ is the free $P(y)$-module generated by monomials $x_{i_{1}} \ldots x_{i_{s}}$. Since all $Q_{i}\left(x_{j}\right) \in P(y)$ from (A.2), we know that $Q_{i}: X_{s} \rightarrow X_{s-1}$, In particular, we can write $x=\sum_{j} u_{j} x_{j}, u_{j} \in P(y)$. Then we have the relation $\left.\left(\bmod \left(I^{2}\right), v_{n+1}, \ldots\right)\right)$

$$
\begin{aligned}
\sum_{j} & u_{j} \sum_{k}\left(v_{k} Q_{k}\left(x_{j}\right)\right)=\sum_{k} v_{k} \sum_{j} u_{j} Q_{k}\left(x_{j}\right) \\
& =\sum_{k} v_{k} Q_{k}\left(\sum_{j} u_{j} x_{j}\right)=\sum_{k} v_{k} Q_{k}(x)=v_{n} y .
\end{aligned}
$$

Thus we can express the relation type $\left(^{*}\right)$ by taking $(1),(2)$ in Lemma 4.3. By the induction on $n$, we can prove the lemma.

From Lemma 3.1. the following corollary is immediate.

Corollary 4.6. If (A.1),(A,2) are satisfied for simply connected Lie group $G_{i}, 1 \leq i \leq n$, then the product $G=G_{1} \times \ldots \times G_{n}$ satisfies Theorem 1.1 in the introduction.

Proof. Let $T_{i}$ be the maximal torus of $G_{i}$. Since $G_{i} / T_{i}$ and $B T_{i}$ are torsion free, we see

$$
B P^{*}(B / T) / \operatorname{Ideal}\left(i^{*}\right) \cong \otimes_{B P^{*}} B P^{*}\left(G_{i} / T_{i}\right) /\left(\operatorname{Ideal}\left(\sum i_{i}^{*}\right)\right)
$$

Hence the result of Lemma 4.4 holds for this $G$.

On the otherhand (A.2) is satisfied also in $H^{*}(G ; Z / p) \cong \otimes H^{*}\left(G_{i} ; \mathbb{Z} / p\right)$. Hence Lemma 4.5 also holds. Thus we get Theorem 1.1 from the $\bmod \left(I^{2}\right)$ version of Lemma 3.1.

\section{SIMPLE GROUPS}

We study simple groups. The simple Lie groups which have $p$-torsion in $H^{*}(G)$ are divided to the following cases.

Case I. The $(G, p)$ are the exceptional Lie groups $\left(G_{2}, 2\right),\left(F_{4}, 2\right),\left(E_{6}, 2\right),\left(F_{4}, 3\right),\left(E_{6}, 3\right),\left(E_{7}, 3\right)$ and $\left(E_{8}, 5\right)$.

Case II. $\left(E_{8}, 3\right)$.

Case III. The cases $\left(E_{7}, 2\right),\left(E_{8}, 2\right)$.

Cases IV. The classical cases $(\operatorname{Spin}(n), 2)$. 
Case I. We at first study $k(1)^{*}(G)$ for the groups $(G, p)$ in Case I. The ordinary mod $p$-cohomology is written

$$
H^{*}(G ; \mathbb{Z} / p) \cong \mathbb{Z} / p[y] /\left(y^{p}\right) \otimes \Lambda\left(x_{1}, x_{2}, \ldots, x_{l}\right)
$$

where $\left|x_{1}\right|=3,\left|x_{2}\right|=2 p+1,|y|=2 p+2, Q_{1} x_{1}=Q_{0} x_{2}=y$. In this case $k(1)^{*}(G)$ is known. Consider the Atiyah-Hirzebruch spectral sequence

$$
E_{2}^{*, *}=H^{*}\left(G ; k(1)^{*}\right) \Longrightarrow k(1)^{*}(G) .
$$

Th only nonzero differential is $d_{2 p-1}(x)=v_{1} \otimes Q_{1}(x)$. Thus we can prove

$$
k(1)^{*}(G) \cong\left(k(1)^{*}[y] /\left(y^{p}, v_{1} y\right) \oplus k(1)^{*}\left\{x y^{p-1}\right\}\right) \otimes \Lambda\left(x_{2}, \ldots, x_{l}\right)
$$

Moreover, $B P^{*}(G)$ is computed in [Y2],

$B P^{*}(G) \cong\left(B P^{*} \otimes P(y) /\left(p y, v_{1} y\right) \oplus B P^{*}\left\{w_{1}, w_{2}\right\} /\left(p w_{1}+v_{1} w_{2}\right) \oplus B P^{*}\left\{x_{1}^{\prime}, x^{\prime \prime}\right\}\right) \otimes \Lambda\left(x_{3}, \ldots x_{l}\right)$.

Here $w_{1}=x_{1} y^{p-1}, w_{2}=x_{2} y^{p-1}, x_{1}^{\prime}=p x_{1}$ and $x^{\prime \prime}=x_{1} x_{2} y^{p-1}$ in the Atiyah-Hirzebruch spectral sequence converging to $B P^{*}(G)$.

Of course this case satisfies $(\mathrm{A}, 1),(\mathrm{A}, 2)$ in $\S 4$. Moreover it is easy proved that Corollary 4.6 holds without modulo $\left(I^{2}\right)$ for all products of Lie groups of Case I.

Theorem 5.1. For the group $(G, p)$ in Case I, we have the isomorphism

$$
\Omega^{*}(G)_{(p)} \cong \Omega^{*}[y] /\left(p y, v_{1} y, y^{p}\right) .
$$

Case II. Let $(G, p)=\left(E_{8}, 3\right)$. The mod 3 cohomology is

$$
H^{*}\left(E_{8} ; \mathbb{Z} / 3\right) \cong \mathbb{Z} / 3\left[y_{8}, y_{20}\right] /\left(y_{8}^{3}, y_{20}^{3}\right) \otimes \Lambda\left(x_{3}, x_{7}, x_{15}, x_{19}, \ldots\right)
$$

where the suffix means its degree,i.e., $\left|y_{8}\right|=8$. The Minor operations are

$$
Q_{1} x_{3}=Q_{0} x_{7}=y_{8}, \quad Q_{2} x_{3}=Q_{1} x_{15}=Q_{0} x_{19}=y_{20}
$$

and $Q_{i} x_{j}=0$ for other $i, j$. From this we have relations,e.g.,

$$
v_{1} y_{8}+v_{2} y_{20}=0, \quad v_{1} y_{20}=0 .
$$

We easily see the conditions $(\mathrm{A}, 1),(\mathrm{A}, 2)$. The $B P^{*}\left(E_{8}\right)$ is computed $([\mathrm{M}],[\mathrm{Y} 3])$. We also get the result without $\bmod \left(I^{2}\right)$, however for $B P^{*}\left(E_{8} \times E_{8}\right)$ I can not show the result without $\bmod \left(I^{2}\right)$.

Theorem 5.2. Then Theorem 1.1 holds for $\left(E_{8}, 3\right)$ without $\bmod \left(I^{2}\right)$, i.e.,

$$
\Omega\left(E_{8}\right)_{(3)} \cong \Omega^{*}\left[y_{8}, y_{20}\right] /\left(3 y_{8}, 3 y_{20}, y_{3}^{3}, y_{20}^{3}, v_{1} y_{8}+v_{2} y_{20}, v_{1} y_{20}\right) .
$$

Case III. $\left(E_{7}, 2\right),\left(E_{8}, 2\right)$.

The mod 2-cohomology of $E_{8}$ is

$$
\begin{gathered}
H^{*}\left(E_{8} ; \mathbb{Z} / 2\right) \cong \mathbb{Z} / 2\left[x_{3}, x_{5}, x_{9}, x_{15}\right] /\left(x_{3}^{16}, x_{5}^{8}, x_{9}^{4}, x_{15}^{2}\right) \otimes \Lambda\left(x_{17}, x_{23}, x_{27}, x_{29}\right), \\
\text { and } H^{*}\left(E_{7} ; \mathbb{Z} / 2\right) \cong H^{*}\left(E_{8} ; \mathbb{Z} / 2\right) /\left(x_{3}^{4}, x_{5}^{4}, x_{15}^{2}, x_{29}\right) .
\end{gathered}
$$

Let us write $y_{2 i}=x_{i}^{2}$ if it is not zero. The $Q_{i}$-actions are completely determined by HuntonMimura-Nishimoto-Schuster. We have for example, [H-M-N-S], [M-N]

$$
\begin{gathered}
Q_{1} x_{3}=Q_{0} x_{5}=y_{6}, \quad Q_{0} x_{9}=y_{10} \quad Q_{1} x_{15}=Q_{0} x_{17}=y_{18}, \\
Q_{2} x_{23}=Q_{1} x_{27}=Q_{0} x_{29}=y_{30} \\
\text { with } \quad 0=Q_{0} x_{3}=Q_{0} x_{15}=Q_{0} x_{27}, \quad 0=Q_{0} x_{23}=Q_{1} x_{23} .
\end{gathered}
$$

(Of course there many other nonzero operations, e.g., $Q_{2} x_{3}=y_{10}, Q_{3} x_{3}=y_{18}$.)

Assumption (A.2) holds except for $Q_{0} x_{23}=Q_{1} x_{23}=0$ but $Q_{2} x_{23}=y_{30}$. 
If there is a relation in $B P^{*}(X) / I^{2}$

$$
v_{n} a_{n}+v_{n+1} a_{n+1}+\ldots=0 \quad a_{n} \neq 0,
$$

then we say it the relation starting with $v_{n} a_{n}$. For example, the relations in $B P^{*}\left(E_{8}\right) / I^{2}$ starting with $2 y_{6}, v_{1} y_{6}$ are

$$
2 y_{6}+v_{2} y_{6}^{2}+v_{3} y_{10}^{2}=0, \quad v_{1} y_{6}+v_{2} y_{10}+v_{3} y_{18}=0 .
$$

Theorem 5.3. The cases $p=2$ and $G=E_{8}, E_{7}$ satify Theorem 1.1 ,e.g.,

$$
\Omega^{*}\left(E_{8}\right) / I^{2} \cong \Omega^{*}\left[y_{6}, y_{10}, y_{18}, y_{30}\right] /\left(y_{6}^{8}, y_{10}^{4}, y_{18}^{2}, y_{30}^{2}, R, I^{2}\right)
$$

where $R$ is generated by relations starting with

$$
2 y_{6}, v_{1} y_{6}, 2 y_{10}, 2 y_{18}, v_{1} y_{18}, 2 y_{30}, v_{1} y_{30}, v_{2} y_{30},
$$

$$
\Omega^{*}\left(E_{7}\right) / I^{2} \cong \Omega^{*}\left(E_{8}\right) /\left(I^{2}, y_{6}^{2}, y_{10}^{2}, y_{30}\right) .
$$

Proof. We will prove only for $G=E_{8}$ but $E_{7}$ is much easier. Note that the product $\mu: G \times G \rightarrow G$ induces the coproduct $\mu^{*}: B P^{*}(G) \rightarrow B P^{*}(G \times G)$, which is not isomorphic to $B P^{*}(G) \otimes_{B P^{*}} B P^{*}(G)$. Since $y_{i}$ is primitive in $H^{*}(G ; \mathbb{Z} / 2)$, we can write in $B P^{*}(G \times G)$

$$
\mu^{*}\left(y_{i}\right)=y_{i} \otimes 1+1 \otimes y_{i}+a \quad \text { with } a \in I=\left(2, v_{1}, . .\right) .
$$

Hence $y_{i}^{2^{k}}, k \geq 1$ is always primitive $\bmod \left(I^{2}\right)$. Conversely $\bmod \left(I^{2}\right)$ primitive elements are of the form $y_{i}^{2 k^{\prime}}, k^{\prime} \geq 0$ and $x_{i}$. By the dimensionl reason, we see $y_{6}^{8}=0, y_{10}^{4}=0, \ldots$

The part which does not satify $(\mathrm{A}, 2)$ is come from

$$
Q_{0} x_{23}=Q_{1} x_{23}=0, \quad Q_{2} x_{23}=y_{30} .
$$

Here we do $k(2)$ version of the proof of Lemma 4.4. Letting $y_{i}=y_{30}$ and $x_{i^{\prime}}=x_{23}$, we can take in $k(2)^{*}(G / T), v_{2} y_{i}=b_{i^{\prime}}+b^{\prime}$ same as $\left(^{*}\right)$ in the proof of Lemma 4.4. All arguments work similarly and we get the theorem.

Case IV ; classical group cases.

We first consider the orthogonal groups $G=S O(m)$, while these are not simply connected. The mod 2-cohomology is written as ( see for example $[\mathrm{N}]$ )

$$
\operatorname{grH}^{*}(S O(m) ; \mathbb{Z} / 2) \cong \Lambda\left(x_{1}, x_{2}, \ldots, x_{m-1}\right)
$$

where the multiplications are given by $x_{s}^{2}=x_{2 s}$. We write $y_{2(o d d)}=x_{o d d}^{2}$. Hence we can write

$$
H^{*}(S O(m) ; \mathbb{Z} / 2) \cong \mathbb{Z} / 2\left[y_{4 i+2} \mid 2 \leq 4 i+2 \leq m-1\right] /\left(y_{2 i+1}^{s(i)}\right) \otimes \Lambda\left(x_{1}, x_{3}, \ldots\right)
$$

where $s(i)$ is the smallest number such that $2^{s(i)}(4 i+2) \geq m$.

The $Q_{i}$-operations are given by Nishimoto $[\mathrm{N}]$

$$
Q_{n} x_{\text {odd }}=x_{o d d+\left|Q_{n}\right|} \cdot \quad Q_{n} x_{\text {even }}=Q_{n} y_{\text {even }}=0 .
$$

We note the operations

$$
Q_{0} x_{1}=y_{2}, \quad Q_{1} x_{2(o d d)-3}=Q_{0} x_{2(o d d)-1}=y_{2(\text { odd })} \text { if }(\text { odd })>1 .
$$

Take new generators $x_{4 m-1}^{\prime}=x_{4 m-1}+x_{2 m-1} y_{2 m}, y_{4 m+2}^{\prime}=y_{4 m+2}+y_{2 m+2} y_{2 m}, x_{4 m+1}^{\prime}=$ $x_{4 m+1}+x_{2 m+1} y_{2 m}$ so that

$$
Q_{0}\left(x_{4 m-1}^{\prime}\right)=0, \quad Q_{1}\left(x_{4 m-1}^{\prime}\right)=Q_{0}\left(x_{4 m+1}^{\prime}\right)=y_{4 m+2}^{\prime} .
$$


Thus we see that the assumption (A.1),(A,2) are satisfied and Theorem 1.1 holds for $G=$ $S O(m)$. Relations are given by

$$
\sum_{n} v_{n} Q_{n}\left(x_{o d d}\right)=\sum_{n} v_{n} x_{o d d+\left|Q_{n}\right|}=0 \quad \bmod \left(I^{2}\right) .
$$

For example, the relations in $B P^{*}(S O(m)) / I^{2}$ starting with $2 y_{6}$ and $v_{1}\left(y_{6}+y_{2}^{3}\right)$

$$
\begin{gathered}
2 y_{6}+v_{1} y_{2}^{3}+v_{2} y_{6}^{2}+v_{3} y_{10}^{2}+\ldots=0 \\
v_{1}\left(y_{6}+y_{2}^{3}\right)+v_{2}\left(y_{10}+y_{2}^{5}\right)+v_{3}\left(y_{18}+y_{2}^{9}\right)+v_{4}\left(y_{34}+y_{2}^{17}\right)+\ldots=0
\end{gathered}
$$

Next consider the case $G=\operatorname{Spin}(m)$. Let $2^{t-1}<m \leq 2^{t}$. Then the mod 2-cohomology is

$$
H^{*}(B \operatorname{Spin}(m) ; \mathbb{Z} / 2) \cong H^{*}(B S O(m) ; \mathbb{Z} / 2) /\left(y_{2}\right) \otimes \Lambda(z)
$$

with $|z|=2^{t}-1$. Here this element $z$ is defined as following. Consider the spectral sequence

$$
E_{2}^{*, *} \cong H^{*}\left(B \mathbb{Z} / 2 ; H^{*}(\operatorname{Spin}(m) ; \mathbb{Z} / 2)\right) \Longrightarrow H^{*}(B S O(m) ; \mathbb{Z} / 2)
$$

induced from the cofibering $\operatorname{Spin}(m) \rightarrow S O(m) \rightarrow B \mathbb{Z} / 2$. Let $x \in H^{1}(B \mathbb{Z} / 2 ; \mathbb{Z} / 2)$ be the genrator. Then $z$ is defined as an element with $d_{2^{t}}(z)=x^{2^{t}}$ since this element is zero in $H^{*}(S O(m) ; \mathbb{Z} / 2)$.

The $Q_{i}$-actions of $z$ are also given by Nishimoto $[\mathrm{N}]$

$$
\begin{gathered}
Q_{0} z=\sum_{i+j=2^{t-1}, i<j} x_{2 i} x_{2 j} \\
Q_{n} z=\sum_{i=2^{n}+1}^{2^{t-2}+2^{n-1}-1} x_{2 i} x_{2^{t}+2^{n+1}-2-2 i} \quad \text { for } n \geq 1
\end{gathered}
$$

It is immediate $Q_{n} z \in P(y)$ and decomposable as a product of generators $x_{\text {even }}$.

Theorem 5.4. The groups $G=S O(m)$, Spin $(m)$ satisfy Theorem 1.1. There are $\Omega_{(2)^{-}}^{*}$ algebra isomorphisms

(1) $\Omega^{*}(S O(m)) / I^{2} \cong \Omega^{*}\left[y_{4 i+2} \mid 2 \leq 4 i+2 \leq m-1\right] /\left(R, y_{4 i+2}^{2^{s(i)}}, I^{2}\right)$

where $R=\left\{\right.$ relations starting with $2 y_{4 i+2}, \quad v_{1}\left(y_{2 i} y_{2 i+2}+y_{4 i+2}\right)$ for $\left.y_{4 j}=y_{2 j}^{2}\right\}$.

(2) $\quad \Omega^{*}\left(\operatorname{Spin}(m) / I^{2} \cong \Omega^{*}(\operatorname{Spin}(m)) /\left(y_{2}, \sum v_{n} Q_{n}(z), I^{2}\right)\right.$

Proof. Let $G=\operatorname{Spin}(m)$. To see the results of Lemma 4.4, Lemma 4.5, we need to see that the relation

$$
\sum v_{n} Q_{n}(z)=0 \quad \text { in } B P^{*}(G) / I^{2}
$$

comes from an element in Ideal $\tilde{B} P^{*}(G / T)$.

Let $b_{z} \in H^{*}(B T ; \mathbb{Z} / 2)$ be the element with $d_{|z|+1}(z)=b_{z}$ in the spectral sequence converging $H^{*}(G / T ; \mathbb{Z} / 2)$. There are many elements $x \in H^{*}(G ; \mathbb{Z} / 2)$ such that $Q_{n} x=Q_{n} z$. Let us write $x=\sum f_{\text {odd }}(y) x_{o d d}$ with $f_{\text {odd }}(y) \in P(y) / 2$. Recall that $Q_{n} z$ is the decomposable element for generators $x_{\text {even }}$ from the result of Nishimoto. Hence we need only consider the cases $\left|f_{\text {odd }}(y)\right|>0$, namely, $\left|x_{\text {odd }}\right|<|z|$. Since $d_{\left|x_{\text {odd }}\right|+1}\left(x_{\text {odd }}\right) \neq 0$, this element disappears in $H^{*}\left(p^{-1}\left(G / T^{\left|b_{z}\right|-1}\right) ; \mathbb{Z} / 2\right)$ from $\left|x_{\text {odd }}\right|+1<|z|+1=\left|b_{z}\right|$.

For each $n$, we consider the spectral sequence

$$
H^{*}\left(B T ; k(n)^{*}(G)\right) \Longrightarrow k(n)^{*}(G / T) .
$$

By the arguments similar to the proof of Lemma 4.3, we get

$$
(*) \quad v_{n} Q_{n}(z)=b_{z}+b_{n}^{\prime} \quad b_{n}^{\prime} \in E_{\infty}^{\left|b_{z}\right|+1, *} .
$$


Moreover the arguments similar to the proof of Lemma 4.5, we can see that $b_{n}^{\prime}=v_{n} b_{n}^{\prime \prime}$ and $b_{n}^{\prime \prime} \in I(t)$.

Let us write

$$
\sum v_{n} Q_{n}(z)-b_{z}=\sum v_{n} a_{n} \quad \text { in } B P^{*}(G / T) / I^{2} .
$$

Considering above equation in $k(n)^{*}(G / T)$, we have $a_{n}=b_{n}^{\prime \prime} \bmod \left(2, v_{1}, \ldots, \hat{v}_{n}, \ldots\right)$. Hence we have $\sum v_{n} Q_{n}(z)+b_{z} \in I(t) \bmod \left(I^{2}\right)$ i.e., the sum $\sum v_{n} Q_{n}(z)$ itself in $I(t)$ modulo $I^{2}$.

From Theorem 5.1- 5.4 and Corollary 4.6, we get Theorem 1.1, and moreover we explicitely know $\Omega^{*}(G) / I^{2}$ for all simply connected Lie groups ( and orthogonal groups).

\section{EXAMPLE FOR THE SPECTRAL SEQUENCES}

In this section, we give examples for spectral sequences for groups $G$ in the most easy case Case I. We consider the spectral sequence

$$
\text { (6.1) } E(h)_{2}^{*, *}=H^{*}\left(B T ; h^{*}(G / T)\right) \Longrightarrow h^{*}(G / T)
$$

for homology theories, $h=H \mathbb{Z} / p, H \mathbb{Z}, H \mathbb{Q}, k(1), K(1), B P\langle 1\rangle$. Since $G / T$ has no torsion, in any $h$ we know that

$$
\text { (6.2) } h^{*}(G / T) \cong h^{*} \otimes P(y) \otimes P^{\prime}(t) /\left(b_{1}, \ldots, b_{l}\right) \quad \text { where } P^{\prime}(t)=\mathbb{Z}\left[t_{1}, \ldots, t_{l}\right] .
$$

First recall the case $h=H Z / p$, the cohomology is $h^{*}(G ; \mathbb{Z} / p) \cong P(y) / p \otimes \Lambda\left(x_{1}, \ldots x_{l}\right)$. The diffirentials are $d_{\left|x_{i}\right|+1}\left(x_{i}\right)=b_{i}$ and we still know

$$
\text { (6.3) } E(H \mathbb{Z} / p)_{\infty}^{*, *} \cong P(y) / p \otimes P(t)^{\prime} /\left(b_{1}, \ldots, b_{l}\right) .
$$

Hereafter we restrict ourself for Case I. This case $P(y) \cong \mathbb{Z}[y] /\left(y^{p}\right)$ and $Q_{1} x_{1}=Q_{0} x_{2}=y$.

Next we consider the spectral sequences for Morava $K$-theories. First recall again

$$
k(1)^{*}(G) \cong\left(P(y)^{+} / p \oplus k(1)^{*} \Lambda\left(x_{1} y^{p-1}\right)\right) \otimes \Lambda\left(x_{2}, \ldots, x_{l}\right) .
$$

We also note that $B P^{*}(G ; \mathbb{Z} / p) \cong B P^{*} \otimes_{k(1)^{*}} k(1)^{*}(G)$ identifying $B P^{*} / p \supset \mathbb{Z} / p\left[v_{1}\right] \cong$ $k(1)^{*}$.

Proposition 6.1. Let $G$ be a simple Lie group in Case I. Then we get isomorphisms

$$
\begin{gathered}
E(k(1))_{\infty}^{* * *} \cong k(1)^{*} \otimes P(y) /\left(v_{1} y\right) \otimes P(t)^{\prime} /\left(b_{2}, \ldots, b_{l}, b_{1}^{s} y^{p-s} \mid 1 \leq s \leq p\right) . \\
E(K(1))_{\infty}^{* * *} \cong K(1)^{*} \otimes P(t)^{\prime} /\left(b_{1}^{p}, b_{2}, \ldots, b_{l}\right) .
\end{gathered}
$$

Proof. First note some facts of the differentials in $K(1)^{*}$-theory. Since $K(1)^{*}(-)$ holds Kunneth formula, we can defined the coalgebra map $\mu^{*}$ on also $E(K(1))_{r}^{*, *}$ considering the fibering

$$
G \times G \rightarrow G \times G / T \rightarrow G \times B T .
$$

Each element $x_{1} y^{p-1}, x_{i}$ are primitive in $K(1)^{*}(G)$, but

$$
\mu^{*} d_{r}\left(x_{i}\right)=d_{r}\left(\mu^{*} x_{i}\right)=d_{r}\left(x_{i} \otimes 1+1 \otimes x_{i}\right)=1 \otimes d_{r}\left(x_{i}\right) .
$$

The differntials images are written as $d_{r}\left(x_{i}\right)=\sum f(x) g(t)$ for $g(t) \in K(1)^{*} P(t)^{\prime}$ and $f(x) \in$ $\Lambda\left(x_{1} y^{p-1}, x_{2}, \ldots, x_{l}\right)$. However if $|f(x)|>0$, then $\mu^{*} d_{r}\left(x_{i}\right) \neq 1 \otimes d_{r}\left(x_{i}\right)$. Thus we get

$$
(*, *) \quad d_{r}\left(x_{i}\right), d_{r}\left(x_{1} y^{p-1}\right) \in K(1)^{*} P(t)^{\prime} .
$$

Now we study the $k(1)^{*}$-theory. Recall $E(k(1))_{2}^{* * *} \cong k(1)^{*}(G) \otimes P(t)^{\prime}$. Suppose $d_{2}(x) \neq 0$.

(1) The case $d_{2}(x)$ is $v_{1}$-torsion.

This case $d_{2}(x)$ is a $k(1)^{*}$-module generator, since $E(k(1))_{2}^{*, *}$ does not has higher $v_{1}$-torsion. Hence $d_{2}(x) \neq 0$ also in $E(H \mathbb{Z} / p)_{2}^{*, *}$ because the Thom map $E(k(1))_{2}^{*, *} /\left(v_{1}\right) \rightarrow E(H \mathbb{Z} / p)_{2}^{*, *}$ is injective. This is a contradiction to $d_{2}(x)=0 \in E(H \mathbb{Z} / p)_{2}^{*, *}$. 
(2) The case $d_{2}(x)$ is in $v_{1}$-image.

Since also $E(k(1))_{2}^{*, *}$ has no higher $v_{1}$-torsion, we see $d_{2}(x) \neq 0$ also in $E(K(1))_{2}^{*, *}$. Let us write $d_{2}(x)=\sum f(x) g(t)$. , Then by dimensional reason, it is neccesary $|f(x)|>0$. This is a contradiction to $(*, *)$. Thus we get $d_{2}(x)=0$.

Next consider $d_{4}(x)$. By the same reasons as $(1),(2)$, we see $d_{4}\left(x_{i}\right)=0$ for $i \neq 1$. By the reason as $(1)$, we see $d_{4}\left(x_{1} y^{p-1}\right)=b_{1} y^{p-1}$. Since $b_{1} y^{p-1}$ is $v_{1}$-torsion, the elemnt $v_{1} x_{1} y^{p-1}$ is a $k(1)^{*}$-module generator in $E(k(1))_{5}^{*, *}$. The differentials are defined as some boundary maps, we get

$$
\partial\left(x_{1} y^{p-1}\right)=b_{1} y^{p-1} \quad \bmod (\text { some filtration })
$$

which implies that

$$
\partial\left(v_{1} x_{1} y^{p-1}\right)=b_{1}\left(v_{1} y\right) y^{p-2}=b_{1}^{2} y^{p-2} \bmod (\text { some filtration })
$$

where we take $y$ such that $v_{1} y=b_{1} \in k(1)^{*}(G / T)$. This follows the fact

$$
d_{2\left|b_{1}\right|}\left(v_{1} x_{1} y^{p-1}\right)=b_{2}^{2} y^{p-2} \quad \bmod (\text { some filtrarion }) .
$$

By induction on $r$ for $d_{r}\left(x_{i}\right)$, we can prove $d_{\left|b_{i}\right|}\left(x_{i}\right)=b_{i}$ for $i \neq 1$, and $d_{s\left|b_{1}\right|}\left(v_{1}^{s-1} x_{1} y^{p-1}\right)=$ $b_{1}^{s} y^{p-s}$ for $1 \leq s \leq p$ by the arguments similar to (1),(2). For $K(1)^{*}$-theory we can prove that $d_{p\left|b_{1}\right|}\left(x_{2} y^{p-1}\right)=v_{1}^{-(p-1)} b_{1}^{p}$ and the result of the proposition.

We consider the integral cohomology $h=H \mathbb{Z}$. Since these groups $G$ has no higher $p$-torsion, we have

$$
\begin{gathered}
H^{*}(G ; \mathbb{Z}) \cong\left(P(y)^{+} /(p y) \oplus \Lambda\left(x_{2} y^{p-1}\right)\right) \otimes \Lambda\left(x_{1}, x_{3}, \ldots, x_{l}\right), \\
H^{*}(G ; \mathbb{Q}) \cong \mathbb{Q} \otimes \Lambda\left(x_{2} y^{p-1}, x_{1}, x_{3}, \ldots, x_{l}\right) .
\end{gathered}
$$

By the arguments similar to the case Morava $K$-theories, but more easily, we get the following differentials.

The differentials in $E(\mathbb{Q})_{r}^{*, *}$ are $d_{b_{i} \mid}\left(x_{i}\right)=b_{i}$ for $i \neq 2$, and $d_{p\left|b_{2}\right|}\left(x_{2} y^{p-1}\right)=b_{2}^{p}$.

The differentials in $E(H \mathbb{Z})_{r}^{*, *}$ are $d_{s\left|b_{2}\right|}\left(p^{s-1} x_{2} y^{p-1}\right)=b_{2}^{s} y^{p-s}$ and $d_{\left|x_{i}\right|+1}\left(x_{i}\right)=b_{i}$ for $i \neq 2$.

Proposition 6.2. Let $G$ be a simple Lie group in Case I. Then we get isomorphism

$$
\begin{gathered}
E(H \mathbb{Z})_{\infty}^{*, *} \cong P(y) /(p y) \otimes P(t)^{\prime} /\left(b_{1}, b_{3}, \ldots, b_{l}, b_{2}^{s} y^{p-s} \mid 1 \leq s \leq p\right), \\
E(H \mathbb{Q})_{\infty}^{*, *} \cong P(t)^{\prime} /\left(b_{1}, b_{2}^{p}, b_{3}, \ldots, b_{l}\right)
\end{gathered}
$$

Now we recall the $B P\langle 1\rangle^{*}$ theory with the coefficient $B P\langle 1\rangle^{*}=\mathbb{Z}_{(p)}\left[v_{1}\right]$ so that $B P\langle 1\rangle^{*} / p-$ $k(1)^{*}$. For $G$ in Case I, it is known that $B P\langle 1\rangle^{*}(G) \cong B P\langle 1\rangle^{*} \otimes_{B P^{*}} B P^{*}(X)$. (See Case I in $§ 5)$. The rational $B P$-theory is immediate

$$
B P^{*}(G ; \mathbb{Q}) \cong B P^{*} \otimes Q \otimes \Lambda\left(x_{1}^{\prime}, w_{2}\right) \otimes \Lambda\left(x_{3}, \ldots, x_{l}\right) .
$$

The differentials in $E(B P \otimes \mathbb{Q})_{r}^{*, *}$ are given by $d_{4} x_{1}^{\prime}=p b_{1}, d w_{2}=b_{2}^{p}$. Here we use notations that $x_{1}^{\prime}, w_{1}, w_{2}$ correspond elemnts $p x_{1}, x_{1} y^{p-1}, x_{2} y^{p-1}$ respectively, in the spectral sequence. ( Recall also Case I in $§ 5$.)

Theorem 6.3. Let $G$ be a simple Lie group in Case I and $E(B P\langle 1\rangle)_{r}^{*, *}$ be the spectral sequence (6.1) for $h=B P\langle 1\rangle$. Then we have the isomorphism

$$
E(B P\langle 1\rangle)_{\infty}^{* * *} \cong B P\langle 1\rangle^{*} \otimes P(y) /\left(p y, v_{1} y\right) \otimes P(t)^{\prime} /\left(p b_{1}, b_{3}, \ldots, b_{l}, b_{1}^{s} b_{2}^{r} y^{p-s-r} \mid 1 \leq s+r \leq p\right)
$$


Proof. Compare this spectral sequence to those of the other theories $k(1), H \mathbb{Z}, B P\langle 1\rangle \otimes \mathbb{Q}$. By induction on $r$ of $d_{r}\left(x_{i}\right)$, we show in $E(B P\langle 1\rangle)_{r}^{*, *}, d_{\left|b_{i}\right|}\left(x_{i}\right)=b_{i}$ for $i \neq 1,2$ and $d_{4}\left(x_{1}^{\prime}\right)=$ $p b_{1}$, and moreover

$$
\begin{aligned}
d_{s\left|b_{1}\right|+r\left|b_{2}\right|}\left(p^{r} v_{1}^{s-1} w_{1}\right) & =b_{1}^{s} b_{2}^{r} y^{p-s-r} \text { for } 1 \leq s+r \leq p \\
d_{r\left|b_{2}\right|}\left(p^{r-1} w_{2}\right) & =b_{2}^{r} y^{p-r} \text { for } 1 \leq r \leq p .
\end{aligned}
$$

Thus we can prove this theorem.

The cohomology $B P^{*}(G / T)$ is still given in (6.2), while it does not give the imformation of $i^{*} B P^{*}(B T)$. However the above theorem says more strong facts. For example

$$
p^{s} v_{1}^{r} y^{p-s-r-1} \notin \operatorname{Im}\left(i^{*}\left(B P^{*}(B T)\right) \quad \bmod \left(v_{2}, \ldots\right), \quad \text { if } s+r<p-1\right.
$$

while of course it is in $\operatorname{Ideal}\left(i^{*}\left(\tilde{B P}^{*}(B T)\right)\right)$. Hence we can know that these facts also hold for $\Omega^{*}(-)$ and $M G L^{2 *, *}(-)$ although they have not the Serre spectral sequences.

\section{MOTIVIC THEORIES}

In this section, we study motivic generalized theories defined by Voevodsky [Vo1],[Vo2]. When $X$ has a cellular decomposition, we can show

$$
h^{*, *}(X) \cong h^{*, *}(p t) \otimes H^{*, *}(X),
$$

for $h^{*, *}(-)$ the generalalized motivic cohomology, e.g., $h=H \mathbb{Z} / p, M G L$. The spaces $X=$ $G / T, B T$ have this property.

We first study the mod $p$ motivic cohomology theory. It is known that there is an element $\tau \in H^{0,1}(X ; \mathbb{Z} / p)$ such that $t_{\mathbb{Z} / p}(\tau)=1$ where $t_{\mathbb{Z} / p}: H^{*, *^{\prime}} \rightarrow H^{*}(X ; \mathbb{Z} / p)$ is the realization map. Recall also $H^{*}(G ; \mathbb{Z} / p) \cong P(y) / p \otimes \Lambda\left(x_{1}, \ldots, x_{l}\right)$. (when $p=2$, we let $y_{i}=x_{i}^{2}$.)

Theorem 7.1. Let $G$ be a simply connected Lie group. Giving bidegree to $H^{*}(G ; \mathbb{Z} / p)$ by $\operatorname{deg}\left(y_{i}\right)=\left(\left|y_{i}\right|,\left|y_{i}\right| / 2\right)$ and $\operatorname{deg}\left(x_{i}\right)=\left(\left|x_{i}\right|,\left(\left|x_{i}\right|+1\right) / 2\right)$, we have the injection

$$
P(y) / p \otimes \Lambda\left(x_{1}, \ldots, x_{l}\right) \otimes \mathbb{Z} / p[\tau] \subset H^{*, *}\left(G_{\mathbb{C}} ; \mathbb{Z} / p\right)
$$

such that for $p=$ odd, it is a ring monomorphism, and for $p=2$, it is a ring monomorphism to $\operatorname{grH}^{*, *}\left(G_{\mathbb{C}} ; \mathbb{Z} / 2\right)$ and $x_{i}^{2}-y_{i} \tau \in \operatorname{Ker}\left(t_{\mathbb{Z} / p}\right)$.

Proof. Consider the commutative diagram

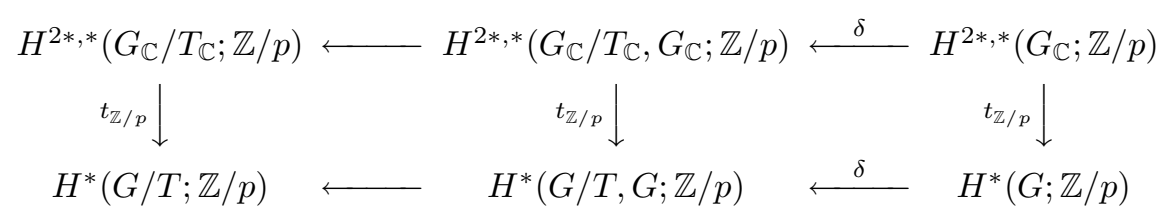

where rows are exact. The cohomology $H^{*}(G / T, G ; \mathbb{Z} / p)$ is computed by using the spectral sequence

$$
E_{2}^{*, *}(G / T, G)=\left(E_{2}^{*, *}-E_{2}^{0, *}\right) \Longrightarrow H^{*}(G / T, G ; \mathbb{Z} / p)
$$

where $E_{2}^{*, *}$ is the spectral sequence converging to $H^{*}(G / T ; \mathbb{Z} / p)$.

Since the differential in $E_{r}^{*, *}$ are given $d_{\left|b_{i}\right|}\left(x_{i}\right)=b_{i}$, we easily seen from the definition of differential, $\delta\left(x_{j}\right)=b_{j} \bmod \left(E_{\infty}^{\left|b_{i}\right|+1, *}\right)$ in $H^{*}(G / T ; \mathbb{Z} / p)$. Here note that $t_{1}, \ldots, t_{l}$ are in $H^{*}(G / T, G ; \mathbb{Z} / p)$, while $y_{i}$ is not in $H^{*}(G / T, G ; \mathbb{Z} / p)$ because $t_{i}=0 \in H^{*}(G ; \mathbb{Z} / p)$. Moreover $\delta\left(x_{i}\right)=b_{i}$ implies $b_{i} \neq 0 \in H^{*}(G / T . G ; \mathbb{Z} / p)$.

Similarly $t_{1}, \ldots, t_{l}$ are in $H^{2 *, *}\left(G / T, G_{\mathbb{C}} ; \mathbb{Z} / p\right)$, since the corresponding elements in $H^{2 *, *}(G / T ; \mathbb{Z} / p)$ goes to zero in $H^{2 *, *}\left(G_{\mathbb{C}} ; \mathbb{Z} / p\right)$. Moreover $b_{i} \neq 0 \in H^{2 *, *}\left(G / T, G_{\mathbb{C}} ; \mathbb{Z} / p\right)$ since it is nonzero in $H^{*}(G / T, G ; \mathbb{Z} / p)$. Hence there is the element $x_{i} \in H^{2 *-1, *}\left(G_{\mathbb{C}} ; \mathbb{Z} / p\right)$ such that $\delta\left(x_{i}\right)=b_{i}$. 
Since $t_{\mathbb{Z} / p}(\tau)=1$, we also see $t_{\mathbb{Z} / p}\left(y_{i} \tau\right)=t_{\mathbb{Z} / p}\left(x_{i}^{2}\right)$ for $p=2$.

The injection in the above theorem seems an isomorphism but I can not prove it now.

Other types of motivic cohomology seem quite complicated and we consider only group $G$ in Case I. By using arguments similar to the $\bmod p$ case, we have ;

Proposition 7.2. Let $G$ be a simple Lie group in Case I. Giving the bidegree by $\operatorname{deg}(y)=$ $(|y|,|y| / 2), \operatorname{deg}(x)=(|x|,(|x|+1) / 2)$ for $x=x_{i}, x_{2} y^{p-1}$, we have the ring monomorphism

$$
H^{*}(G) \cong\left(\mathbb{Z}[y] /\left(p y, y^{p}\right) \oplus \mathbb{Z}\left\{x_{2} y^{p-1}\right\}\right) \otimes \Lambda\left(x_{1}, x_{3}, \ldots, x_{l}\right) \subset H^{*, *}\left(G_{\mathbb{C}}\right) .
$$

Recall the motivic cobortdism $M G L^{*, *}\left(G_{\mathbb{C}}\right)$ defined by Voevodsky. Let $M G L / p^{*, *}(-)$ the motivic cohohomology theory defined by the cofiber sequence of stable $\mathbb{A}^{1}$-homotopy category

$$
M G L \stackrel{p}{\longrightarrow} M G L \longrightarrow M G L / p
$$

so that we have the long exact sequence

$$
\longrightarrow M G L^{*, *}(X) \stackrel{p}{\longrightarrow} M G L^{*, *}(X) \longrightarrow M G L / p^{*, *}(X) \longrightarrow \ldots
$$

Theorem 7.3. Let $G$ be a simple Lie group in Case I. Giving the bidegree by deg $(y)=$ $(|y|,|y| / 2), \operatorname{deg}(x)=(|x|,(|x|+1) / 2)$ for $x=x_{i}, x_{1} y^{p-1}$, we have the $M U^{*} / p$-algebra injection

$$
M U / p^{*}(G) \cong\left(M U^{*} / p \otimes\left(\mathbb{Z}[y] /\left(y^{p}, v_{1} y\right) \oplus \mathbb{Z}\left\{x_{1} y^{p-1}\right\}\right) \otimes \Lambda\left(x_{2}, \ldots, x_{l}\right) \subset M G L / p^{* * *}\left(G_{\mathbb{C}}\right) .\right.
$$

Proof. The proof is quite similar to the case of ordinary mod $p$ motivic cohomology. Here we use the realization map

$$
M G L / p^{* * *}\left(G_{\mathbb{C}} \stackrel{t_{M G L}}{\longrightarrow} M U^{*}(G ; \mathbb{Z} / p) \rightarrow B P^{*}(G ; \mathbb{Z} / p) \rightarrow k(1)^{*}(G)\right.
$$

and the isomorphism $B P^{*}(G ; \mathbb{Z} / p) \cong B P^{*} \otimes_{k(1) *} k(1)^{*}(G)$.

\section{Classifying spaces}

Here we consider the other types of algebraic spaces. For an algebraic group $G$ (not assumed here the connectness) over $\mathbb{C}$, we can construct the classifying space $B G$ as a limit of smooth algebraic varieties. (Indeed we still considered BT.) B.Totaro ([To1],[To2]) first studied the Chow ring of $B G$. He computed most important cases and conjectured $C H^{*}(B G) \cong M U^{*}(B G) \otimes_{M U^{*}} \mathbb{Z}$. He first found the modified cycle map ([To1])

$$
\overline{c l}: C H^{*}(X) \rightarrow M U^{*}(X) \otimes_{M U^{*}} \mathbb{Z}
$$

such that its compostion with the Thom map is the usual cycle map. Now this map is extended by Levine and Morel as the map

$$
t_{M U} \rho_{M G L}: \Omega^{*}(X) \rightarrow M U^{*}(X)
$$

By results of Totaro, the map (7.2) are isomorphism for products of $X=B G L_{n}, B O(n), B Z / p^{n}$, and hence groups whose $p$-Sylow subgroups are abelian $p$-groups ([To1],[To2]). Moreover it is known that (7.1) is epic ,for example, $G=P G L_{3}, S O(4), \operatorname{Spin}(m), m \leq 9, G_{2}$ and the extraspecial $p$ groups of order $p^{3}$ ([Pa], [Ve].[S-Y],[Y4]). Hence all these cases (7. 2) are epic. Thus (7.2) seems to be isomorphism for each $X=B G$. 


\section{REFERENCES}

[G] A. Grothendieck. Torsion homologique et sections rationnelles. Sem. C.Chevalley, ENS 1958, expose 5, Secreatariat Math., IHP, Paris (1958).

[H-M-N-S] J. Hunton, M. Mimura, T. Nishimoto and B. Schuster. Higher $v_{n}$ torsion in Lie groups. J.Mth.Soc.Japan 50 (1998), 801-818.

[I-K-T] K. Ishitoya, A. Kono and H. Toda Hopf algebra structure of mod 2 cohomology of simple Lie groups. Puble. RIMS Kyoto Univ 12 (1976-77), 141-167.

[K] V. G. Kac. Torsion in cohomology of compact Lie groups and Chow rings of reductive algebraic groups. Invent. Math. 80 (1985), 69-79.

[L-M 1] M. Levine and F. Morel. Coborsime algébrique I. C. R. Acad. Sci. Paris 332 (2001), 1-6.

[L-M 2] M. Levine and F. Morel. Coborsime algébrique II. C. R. Acad. Sci. Paris 332 (2001), 815-820.

$[\mathrm{M}] \quad$ M. Mimura. Homotopy theory of Lie groups. Hand book of algebraic topology, Edited by I. James, Elsevier Science BiV. (1995), 951-991.

[M-N] M. Mimura and T. Nishimoto. Hopf algebra structure of Morava K-theory of exceptional Lie groups. To appear. (2001).

[M-V] F. Morel and V. Voevodsky. $\mathbb{A}^{1}$-homotopy theory of schemes. IHES Publ. Math. 90 (2001), 45-143.

$[\mathrm{Ni}] \quad$ T. Nishimoto Higher torsion in Morava K-thoeory of $S O(m)$ and $\operatorname{Sin}(m)$. J.Math.Soc. Japan. 52 (2001), 383-394.

[No] P. Novikov The methods of algebraic topology from the view point of cobordism theory. Math. USSR. Izv. 1 (1967), 827-913.

[P] R. Pandharipande. Equivariant Chow rings of $O(k), S O(2 k+1)$, and $S O(4)$. J. Reine Angew. Math. 496 (1998) 131-148.

[Q] D. Quillen. Elementary proofs of some results of cobordism theory using Steenrod operations. Adv. Math. 7 (1971), 29-56.

[Sc-Y] B. Schuster and N. Yagita. Transfer of Chern classes in BP-cohomology and Cow rings. Trans. AMS. 353 (2001), 1039-1054.

[To1] B. Totaro. Torsion algebraic cycles and complex cobordism. J. Amer. Math. Soc. 10 (1997), 467493.

[To2] B. Totaro. The Chow ring of classifying spaces. Proc.of Symposia in Pure Math. "Algebraic Ktheory" (1997:University of Washington,Seattle) 67 (1999), 248-281.

[Ve] G.Vezzosi. On the Chow ring of the classifying stack of $P G L_{3, C}$. J.Reine Angew. Math. 523 (2000), 1-54.

[Vo1] V. Voevodsky. The Milnor conjecture. Preprint (1996).

[Vo2] V. Voevodsky (Noted by Weibel). Voevodosky's Seattle lectures : $K$-theory and motivic cohomology Proc.of Symposia in Pure Math. "Algebraic K-theory" (199\%:University of Washington, Seattle) 67 (1999), 283-303.

[Y1] N. Yagita. On relations between Brown-Peterson cohomology and the ordinary mod $p$ cohomology theory. Kodai Math.J 7 (1984), 273-285.

[Y2] N. Yagita. Brown-Peterson cohomology groups of exceptional Lie groups. J. Pure and Apllied algebra. 17, (1980) 223-226.

[Y3] N. Yagita. On mod odd prime Brown-Peterson cohomology groups of exceptional Lie groups. J. Math. Soc. Japan. 34, (1982), 293-305.

[Y4] N. Yagita. Chow ring of classifying spaces of extraspecial p-groups. To appear. (2000).

Department of Mathematics, Faculty of Education, Ibaraki University, Mito, Ibaraki, Japan

E-mail address: yagita@mito.ipc.ibaraki.ac.jp 